\title{
Towards Emotional Characters in Computer Games
}

\author{
Robert Schaap and Rafael Bidarra \\ Delft University of Technology \\ Faculty of Electrical Engineering, Mathematics and Computer Science \\ P.O. Box 5031 \\ 2600 GA Delft, The Netherlands \\ R.E.J.Schaap@gmail.com, \\ R.Bidarra@ewi.tudelft.nl
}

\begin{abstract}
This paper describes our research on integrating emotion aspects into characters of video games. Motivation for this is the significant absence of emotive expression in most characters of current games. In order to provide players with a more immersed game experience, it is essential that characters show compelling expressions, in particular, facial expressions. As a research methodology, we took several intense scenes from classic movies and evaluated what would be necessary to allow a virtual character to reenact the scenes inside a game. The recreated scenes should convey the same emotions as the original scenes. Our approach represents the internal state of a video game characters by using a PAD-based model, as well as modeling the impact on it of many relevant environment aspects. Representing this internal state also involves blending together animations to create lively facial expressions. We conclude that the resulting model is good enough to allow scenes to be recreated with an emotional load similar to the original scenes. The approach is also successful inimproving the consistency of the mood of the characters based on their personality.
\end{abstract}

\section{Introduction}

Recently there have been large improvements regarding video games. The advancements in graphics are especially large. Character expression is not getting the attention it deserves to get. Generating interesting and appropriate facial expressions for video game characters is difficult, but it is very important for the overall game-play experience. Most game developers rely heavily on scripting to create the experience they envision. With the expanding scope of current games it becomes more difficult to rely on scripting solely. Game worlds are becoming larger and more complicated. A lot of interaction takes place not just between the players and the game world but also among players and among elements of the game world. Even when we only consider single player games, the game worlds of current games are so vast that it has become very hard to determine when certain events will take place. Even worse, it is often not even possible to anticipate which events will be taking place.

Imagine the situation where the best friend of the player gets shot and right after that something positive happens, so that the latter event would possibly trigger a 


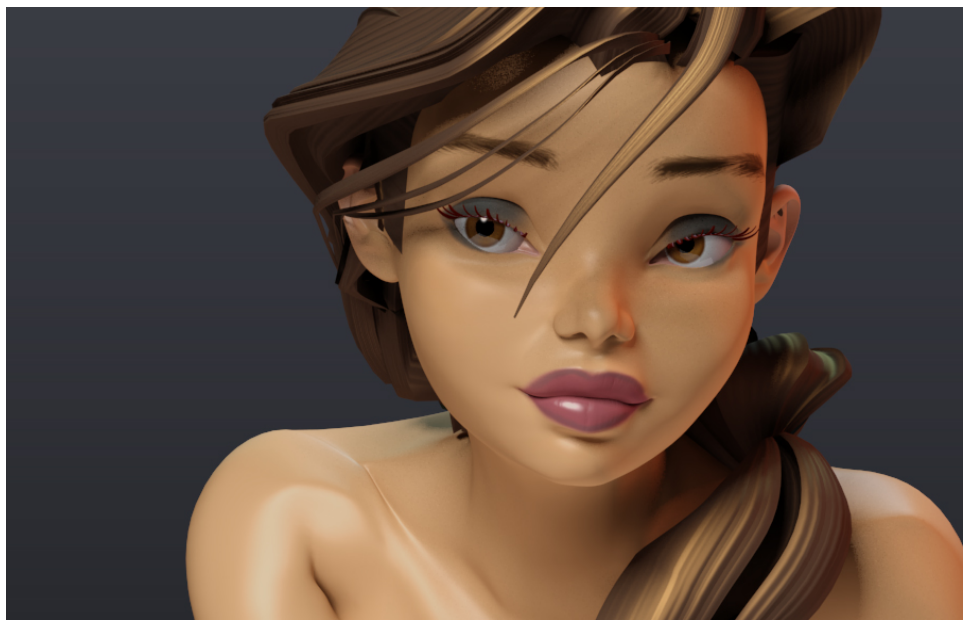

Fig. 1. The Girl character

script for the player to act happy. This would be very awkward and unrealistic since he just lost a good friend. Of course this could be solved with additional scripting, but this clearly does not scale. The scripts will become complicated and still there will always be situations and sequences of events that the developer did not think about.

With this in mind, we investigated ways to make it possible to create more interesting and emotionally convincing video game characters, as that shown in Fig. 1. To accomplish this, we propose a model that is able to represent the internal state of the characters.

Another goal is to assist the artists who create the virtual worlds and define its inhabitants. Artists do not tend to be very technical people, yet a lot of tools out there are very technical and not very intuitive to use. We want to provide them with easyto-use tools for defining personality in video game characters. As well as providing them with a straight-forward way of integrating the characters into their environment. To accomplish this we have taken a highly procedural approach which allows for intuitive parameterizations.

In the next section we will introduce a model that is able to represent personality, mood and emotions. We will determine and structure the environmental influences that affect internal state in section 3. Finally, to bring the characters to life, we need to generate actual expressions for the characters based on their internal state. This is detailed in section 4 , followed by our conclusions and recommendations in section 5 .

\section{Representing Internal State}

The internal state of a character can be divided into three layers: emotion, mood and personality [1] for which the duration of the effect respectively short, medium and long. See Fig. 2 for a visualization of how the three states relate over time. The PleasureArousal-Dominance (PAD) model is a model that has been developed for convenient assessment of consumer reactions to services and products. This means that the model was meant to describe and record emotions. However, after reviewing the model it 


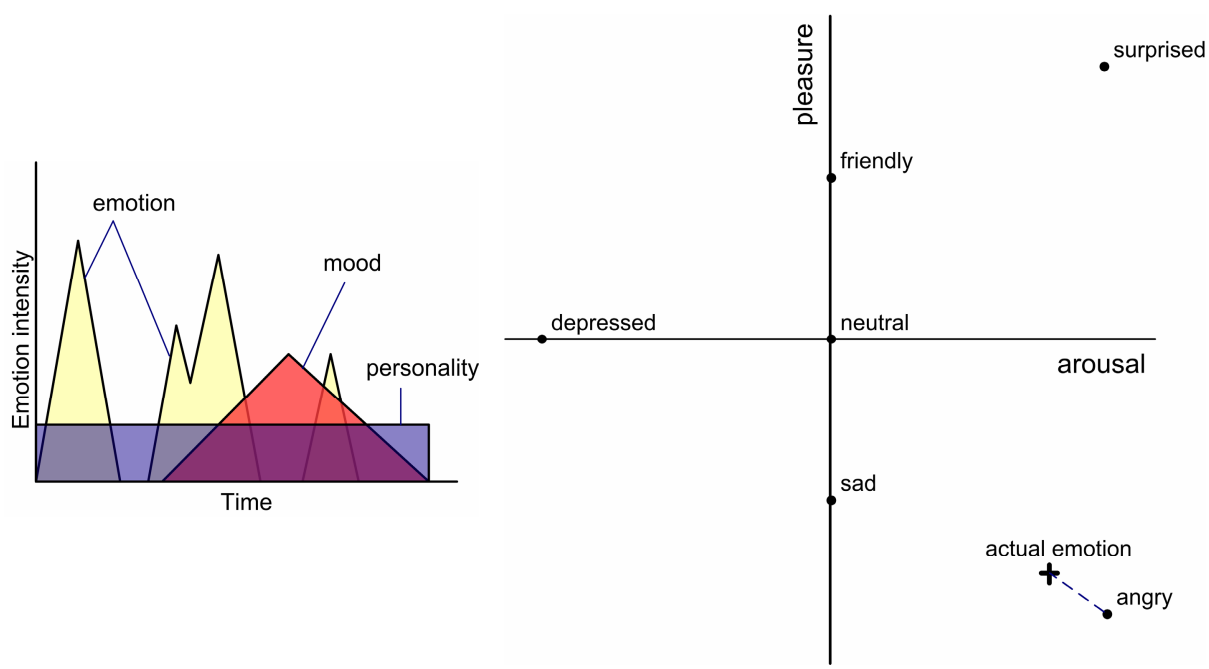

Fig. 2. Emotional intensities over time

Fig. 3. Emotions in PAD space

became clear that it could also be used for tracking the internal state of characters and provide us with a very good basis for generating facial expressions. We have evaluated the PAD model and compared it to alternative models. In this section we point out the strengths of the PAD model, by which we think it is suitable for use in video games.

The PAD model is based on the view of human emotions as an input-output system [2]. The PAD model lies in between these inputs and outputs. It consists of only three emotional dimensions. These dimensions are Pleasure, Arousal and Dominance. The PAD model has a few properties which make it suitable for representing the internal state of characters. The model has a low complexity due to the fact that it uses only three axes for representing emotions. The PAD model is able to represent a broad range of emotions. It can be compared to creating a whole spectrum of colors using only red, green and blue. The interesting thing about the scales is that they are almost orthogonal to each other. This gives us great flexibility as it allows us to modify the values along the three scales independently without trouble. Fig. 3 shows how a set of emotions can be placed inside the PAD model. For illustration purposes, we left out the dominance axis in this figure, resulting in a crosscut of the actual PAD space.

The pleasure scale signifies the amount of enjoyment or satisfaction the character is experiencing. When the character experiences something good he will feel happy, when something bad happens he will be sad. The arousal scale relates to the amount of arousal or excitement being experienced. For instance, when a character is receiving a lot of information he will be aroused; when there is no information he will be bored. The final scale is the dominance scale which relates to the feeling of being incontrol and free. When a character is enslaved, his dominance will be low, when he is free to do what he pleases, his dominance will be high.

Finally, there exist mappings between the PAD model and other well-known models such as Big Five [3]. We use this to provide artists with a set of sliders to tweak personality of characters without having to know about the PAD model. 
Another model that is based on the PAD model is ALMA, in which moods are simulated by using a pull-and-push mood change function [4]. We have taken this idea and applied it to our model, so that we can change the current mood based on the active emotion. When the intensity of the active emotion is higher than the intensity of the current mood the current mood gets pulled towards the active emotion. This results in the amplification of the current mood. When the intensity of the active emotion is lower than the intensity of the current mood the current mood gets pushed away from the active emotion. The intensity of the active emotion is used to determine how hard to push or pull the current mood.

After establishing a model for the internal state we created the means to influence this state from the environment by determining the environmental influences.

\section{Determining Environmental Influences}

Human beings are strongly influenced by their environment. Sometimes they are aware of these influences, but most of the time they do not realize that. Typically, only when these influences become more extreme, people will become aware of them; e.g. extreme heat will certainly be noticed, but a pleasant temperature will not.

Current computer games try to provide gamers with a richer gaming experience. They do this by implementing things like dynamic weather systems and day/night cycles. However, most of the time the characters in these virtual worlds are blissfully unaware of what is happening around them. This makes them seem disconnected from their environment and renders their behavior unrealistic. To fix this we have created a model that takes the effect of the environmental influences into account when determining the internal state of a character.

We started with a survey of influences that are common to characters in computer games. We came to the conclusion we need three basic categories of influences.

These categories are: physical, chemical and psychological influences.

The physical influences come from the body of the character and influence the mood and emotional state of the character directly (e.g. fatigue, illness and hunger). The chemical influences are physical of nature as well, but can be directly linked to substances the character has been exposed to (e.g. alcohol, medicine and drugs). The psychological influences depend on factors that are present in the environment of the character (e.g. freedom, hostility and familiarity). Each of these actual influences has a specific effect on the internal state of the character. For example, fatigue will have a negative effect on the feeling of pleasure and arousal. Alcohol will have a positive effect on pleasure and dominance, but a negative effect on arousal. To accurately model the effects of some influences we needed PAD values that vary over time. Take drugs for example: the way the effects of drugs develop is usually a fast increase right after the drugs have been taken, followed by a slow decay as the drugs are wearing out. New influences can be defined as needed as long as the environmental effects can be expressed in changes through the PAD space.

Now that we have described the internal state and the influences upon it, we have to look at how we are going to make these emotions perceivable to the gamer. 


\section{Generating Expressions from Internal State}

Eyes are a very important part of conveying the internal state of a character. When a character is looking at some items or when he is specifically avoiding them, that says a lot about how the character feels about those items. The way we incorporated the gaze into our model is by relating the arousal scale to the amount of movement of the eyes. A character, who is very aroused, wants to take in as much information as possible and will be looking at many things, frequently switching focus. We also take into account the amount of interest the character has in the objects around him. So he will more frequently and for longer periods of time watch objects that have a higher interest value. On the opposite side, when a character is very non-aroused, he will not look at objects that much. He will stare into empty space, at his feet or at the floor and he will not switch focus very often. The dominance scale will be used for when a character is watching another character. When the value on this scale is high, he or she will look the other character straight in the eyes. When the value is low, he or she will act shy, avoid eye contact and generally look in a downward direction.

Another important part of conveying emotion is the expression on the face of a character. We use the PAD model to determine which emotion will be displayed on the face. In PAD space we have defined a set of emotions (see Fig. 3). For these emotions we have defined actual facial expressions. The actual emotion for a character is marked with a plus-sign. To determine which facial expression should be displayed we take the emotions that are closest to the actual emotion and blend them together using the distance of the actual emotion to the preset emotion as a weight. This means when the actual emotion is not too far from the anger emotion, the character will show a hint of anger. When the actual emotion gets pushed more towards the anger emotion, the intensity of the anger expression on the face will increase. The usual approach in facial animation is to blend together static facial expressions; our approach differs in that we actually blend together facial animations.

Additionally, we add noise to the animations, significantly improving character expression. Even in rest, it is very unnatural for a face of a person to be completely still. The arousal scale of the PAD model is used to determine how much noise to add to the facial animation. Higher values of arousal will result in more and larger movements in the face. This is because a high value for arousal indicates that the character is very excited about something. Blinking is also controlled by the arousal scale. It controls the speed and the frequency of the blinking in much the same way as it controls the added noise. A very aroused person will open his eyes faster and blink more often, whereas eyes that are opening and closing very slow will make a person look very bored or sleepy. All of this makes the characters come better to life.

\section{Conclusions and Future Work}

The use of the PAD model provides a simple yet efficient way of keeping track of the internal state of video game characters. It is useful for maintaining the mood of characters while taking the personality and the current emotions into account. The categorization of environmental influences gives artists an intuitive parameterization, providing a lot of control over how the environment affects the characters. Generating expressions from PAD values seems to work very well for conveying the mood of a 
character. The addition of noise based on PAD values definitely makes the characters more interesting and lifelike.

When using our model for conveying really specific emotions, some information is lost when converting these emotions to PAD values and the results are not always very accurate. This is due to the fact that the PAD model consists of only three dimensions, which can result in the loss of some subtleties in emotions. These subtleties could possibly still be brought back with some tweaking or taken into account by extending our model, but this still needs to be researched further. In addition, we will continue to fine-tune and evaluate our model using the prototype we have developed, and to incorporate new ideas with our game industry partners.

For evaluation purposes we have taken some intense classic movie sequences from movies such as "Crouching Tiger Hidden Dragon", "Pirates of the Caribbean", "Der Untergang" and "Das Boot". These scenes have then been replicated using the system we have developed to enhance the animation and to really bring the characters to life. The scenes we take from these movies contain highly expressive characters. One such example is the scene from "Das Boot" in which a submarine crew member completely loses his sanity during an attack on the ship with depth-charges. Due to strong space limitations we refer the reader to [5].

Even though evaluation is still in progress, we can already conclude that the use of the PAD model as the basis for maintaining mood and emotions, as well as for representing them through facial expressions of game characters, is very promising.

\section{Acknowledgements}

Thanks to Kim Goossens, Jeroen Krebbers and Igor Frid, of Dutch game development studio Playlogic Game Factory, for watching over the direction of this research project from their game development point of view. We also thank Kim for providing us with the (beautiful) Girl character to work with (Fig. 1). Finally we thank all other people at Playlogic Game Factory for their support.

\section{References}

1. Moore, G.: Talking heads: Facial animation in the getaway (2001) (retrieved, February 2006),

http: //www.gamasutra.com/features/20010418/moore_01.htm

2. Mehrabian, A.: The three dimensions of emotional reaction. Psychology Today, 57-61 (1976)

3. John, O.P., Srivastava, S.: The big-five trait taxonomy: History, measurement, and theoretical perspectives. In: Handbook of Personality: Theory and Research. Guilford, New York (1999)

4. Gebhard, P.: ALMA: A Layered Model of Affect. In: AAMAS 2005: Proceedings of the fourth international joint conference on Autonomous agents and multiagent systems, pp. 29-36. ACM Press, New York (2005)

5. Schaap, R.: The PAD model as basis for emotion of video game characters, MSc Thesis, Delft University of Technology, Delft, The Netherlands (2008) 